\title{
LYSOPHOSPHATIDIC ACID-FUNCTIONALISED TITANIUM AS A SUPERIOR SURFACE FOR SUPPORTING HUMAN OSTEOBLAST (MG63) MATURATION
}

\author{
Jason P. Mansell ${ }^{*}$, Judith Brown², Jonathan G. Knapp², Charl F. J. Faul², Ashley W. Blom ${ }^{1}$ \\ ${ }^{1}$ Musculoskeletal Research Unit, Avon Orthopaedic Centre, School of Clinical Sciences, University of Bristol, \\ Westbury-on-Trym, UK \\ ${ }^{2}$ School of Chemistry, University of Bristol, Bristol, UK
}

\begin{abstract}
Covalent modifications of titanium with small molecules known to promote human osteoblast maturation are especially attractive in developing superior biomaterials. An important step in securing competent bone formation at implant sites is promoting the formation of mature osteoblasts, either from committed pre-osteoblasts or from their mesenchymal progenitors. To this end our research has focussed on identifying molecules that enhance human osteoblast formation and maturation and to develop ways of covalently attaching these molecules to implant surfaces so that they are more likely to withstand the rigors of the implantation process whilst still retaining their bioactivity. Herein we report the novel production of lipid-functionalised titanium using lysophosphatidic acid or a related compound, (3S) 1-fluoro-3-hydroxy-4-butyl1-phosphonate. Both lipids were especially effective at co-operating with calcitriol to promote human osteoblast maturation at these modified Ti surfaces in vitro. The novel findings presented offer enticing new developments towards the fabrication of next-generation implant devices with the potential to significantly enhance the osseointegration process and with it improvements in future prosthesis performance and longevity.
\end{abstract}

Keywords: Titanium; surface functionalisation; covalent attachment; lysophosphatidic acid; receptor agonist; active vitamin $\mathrm{D}$; osteoblast maturation.

*Address for correspondence:

Dr. Jason P. Mansell

Musculoskeletal Research Unit

Avon Orthopaedic Centre

School of Clinical Sciences

University of Bristol

Westbury-on-Trym

BS10 5NB, UK.

Tel: +44 1173235966

Fax: +44 1173235936

e-mail: j.p.mansell@bris.ac.uk

\section{Introduction}

Osteoblast formation and maturation at prosthetic surfaces is predicted to enhance biomaterial integration and ultimately implant longevity. The current baseline cost, i.e., the sum provided to the UK's National Health Service to conduct a single joint replacement, is $\sim £ 5$.8k for hips and $\sim £ 5.5 \mathrm{k}$ for knees. The UK alone replaces around 160,000 total hip and knee replacements each year and 10 $\%$ of these prostheses will fail through aseptic loosening. The costs incurred for surgical revision of these failed prostheses is greater still and is predicted to increase as the demand for total joint replacements rises. Indeed recent evidence from the US predicts a $170 \%$ increase in total hip replacement and a $600 \%$ increase in total knee replacement by 2030 (Kurtz et al., 2007). Consequently there continues to be a global incentive to improve implant longevity and this could include steps to biofunctionalise titanium (Ti) with small bioactive molecules.

Realising the fabrication of superior implant materials includes identifying molecules that are reported to promote human osteoblast development and differentiation at implant surfaces. Closely associated with identifying suitable bioactives, are identifying appropriate strategies for attachment of selected molecules to the implant material, whilst, importantly, retaining their biological activity. Although non-covalent steps have been taken to bolster the performance of titanium (Beutner et al., 2010; Hanawa, 2011), it is our opinion that the suitability of such measures to enhance biomaterial functionality in high-performance environments, such as total joint arthroplasty, is unlikely to succeed. Our reasoning is largely pragmatic; selected molecules are more likely to remain tethered to the biomaterial surface and withstand the rigors of implantation if bonded covalently. Another key consideration is size; smaller molecules will exhibit larger surface coverage (especially for porous materials) than much larger polypeptide/protein growth factors, e.g., bone morphogenic proteins (BMPs), and they are less likely to pose steric hindrances to target cell surface receptors.

Recently, we reported that lysophosphatidic acid (LPA) and the LPA $1 / 3$ receptor agonist, (2S)-OMPT, synergistically co-operated with calcitriol (D3) to promote human MG63 osteoblast maturation at both titanium (Ti) and hydroxyapatite (HA) surfaces (Mansell et al., 2009a, 2010). LPA and related lipids, lysophosphatidylcholine (LPC), sphingosine-1-phosphate (S1P) and the structurally related S1P receptor agonist prodrug, FTY720 (Fingolimod), are assuming a growing significance in mineralising tissue biology (Blackburn and 
Fig. 1. A summary of the chemistries employed to covalently tether LPA to titanium. Schematic representation of the initial four routes chosen to functionalise titanium with LPA. The ability of these different surfaces to promote D3-induced human osteoblast maturation was subsequently investigated.

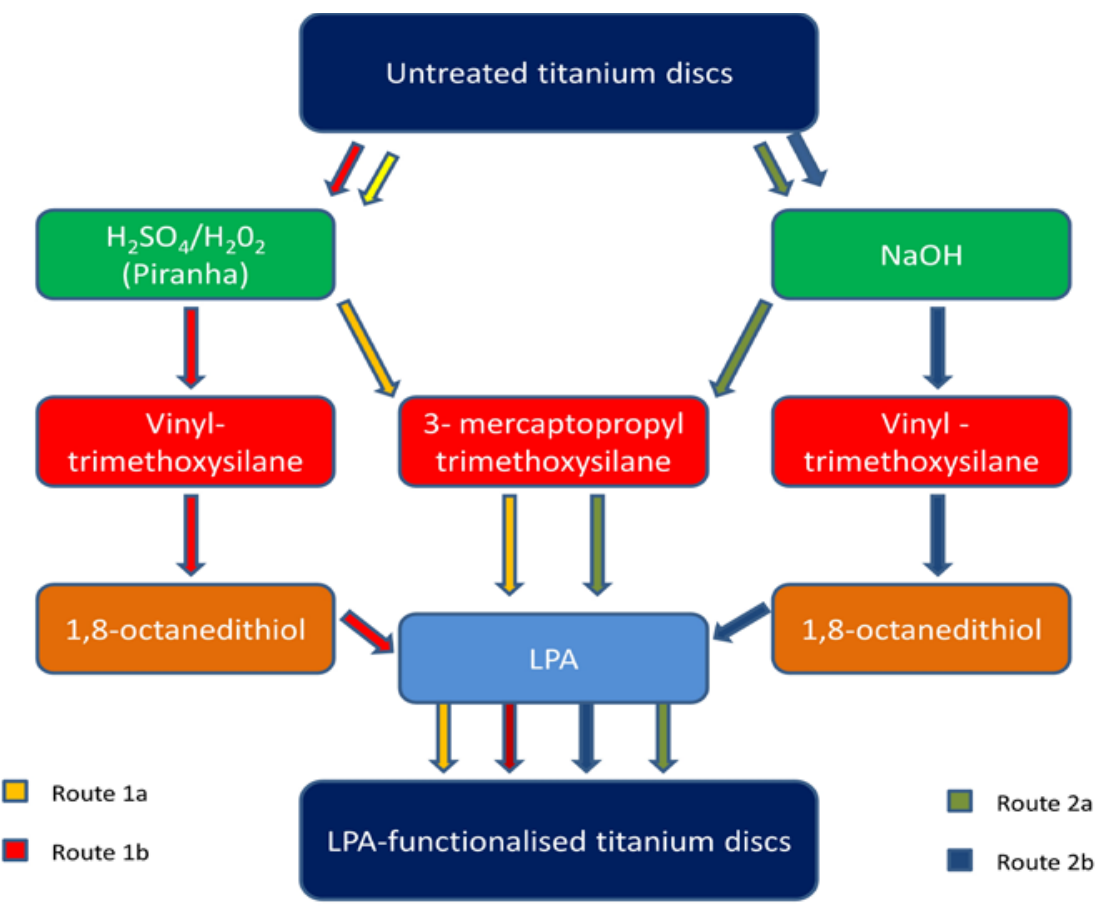

Mansell, 2012); LPC is a major circulating lipid precursor to LPA that has recently been reported to induce the transdifferentiation of human aortic smooth muscle cells to the osteoblast phenotype (Vickers et al., 2010). Both LPA and S1P have been identified as stimulating both human osteoblasts (Dziak et al., 2003; Gidley et al., 2006; Grey et al., 2002; Mansell et al., 2009b; Mansell et al., 2010) and their bone marrow progenitors. It has been proposed that these lipids likely aid cell migration and survival, key events to securing tissue repair/regeneration (P'ebay et al., 2007). Furthermore, recent studies have now revealed that FTY720, a prodrug licensed for treating relapsing forms of multiple sclerosis (Brinkmann et al., 2010; Choi et al., 2011), is capable of securing superior neovascularisation, mechanical stability and osseointegration of large tibial bone allografts in rats (Aronin et al., 2010a). The same compound has also been reported to aid cranial bone defect repair via the recruitment of local bone progenitors (Aronin et al., 2010b). It would appear therefore that certain low molecular mass lipids are attractive candidates in a bone regenerative setting.

Given our discovery that LPA and D3 co-operate to stimulate human osteoblast differentiation (Gidley et $a l .$, 2006), the development of an LPA-functionalised Ti surface seemed a logical next step as part of our on-going programme of research aimed at enhancing the experience of arthroplasty. In this regard, an LPA-functionalised Ti surface would co-operate with local concentrations of D3 to heighten the maturation potential of attached osteoblasts and present a greater potential to enhance initial implant integration into host tissue. In developing an LPA-functionalised Ti surface it is important to ensure the correct alignment of LPA molecules in relation to the metal surface; phospholipids and phosphopeptides have a natural affinity for Ti (Ikeguchi et al., 2000; Larsen et al., 2005) and this non-covalent interaction has been exploited in the fabrication, for example, of solid phase extraction systems to remove phospholipids from complex biological samples, including foodstuffs, e.g., chocolate milkshake (Calvano et al., 2009). However, this phospholipid-Ti interaction is via the polar head group and such a configuration is unsuitable in the generation of LPA-signalling supports, as it is the phosphate group that binds to cell surface receptors to initiate cell signalling (Tigyi and Parrill, 2003). To ensure that LPA is appropriately bound, we targeted the alkene moiety in the tail section for covalent attachment to the metal surface. Herein we describe how the covalent attachment of either LPA or a recently developed LPA1/3 receptor agonist, (3S) 1-fluoro-3-hydroxy-4-butyl-1phosphonate (FHBP), produces a Ti surface that supports superior human osteoblast maturation to D3. Of the materials produced in our laboratory, FHBP-functionalised Ti would appear to be the most promising substrate at supporting D3-induced human osteoblast maturation.

\section{Materials and Methods}

Lipid functionalisation of orthopaedic grade titanium Solid titanium discs $(12.7 \mathrm{~mm}$ diameter and $2.5 \mathrm{~mm}$ thickness) were a generous gift from DePuy (Blackpool, UK), whereas Ti foam discs (12 $\mathrm{mm}$ diameter and $2 \mathrm{~mm}$ thickness) were provided by Corin (Cirencester, UK). These latter samples had a porosity of $514 \mathrm{mg} / \mathrm{mm}^{3} \pm 66$ $\mathrm{mg} / \mathrm{mm}^{3}$ (as sourced from a random batch of 12 samples). A summary of some biomechanical and surface roughness data (as provided by Corin) for these porous samples are provided (Table 1). Unless stated otherwise, all reagents were of analytical grade from Sigma-Aldrich (Poole, UK). Stocks of LPA (Enzo Life Sciences, Exeter, UK) and FHBP (Tebu-bio, Peterborough, UK) were prepared in 1:1 ethanol:tissue culture grade water to a final concentration of $10 \mathrm{mM}$ and stored at $-20{ }^{\circ} \mathrm{C}$. Likewise, stocks of D3 $(100 \mu \mathrm{M})$ were prepared in ethanol and stored at $-20^{\circ} \mathrm{C}$. 
Table 1. A summary of some biomaterial properties of the titanium foam used in this study.

\begin{tabular}{llll} 
Parameter & Units & Mean $^{1}$ & Standard deviation \\
\hline Static shear strength & PSI & 5314.9 & 609.3 \\
Static tensile strength & PSI & 7653.9 & 1403.8 \\
Shear fatigue strength & PSI & 4333.8 & 800.4 \\
\hline
\end{tabular}

${ }^{1}$ The data presented were obtained from a batch of five foam samples.
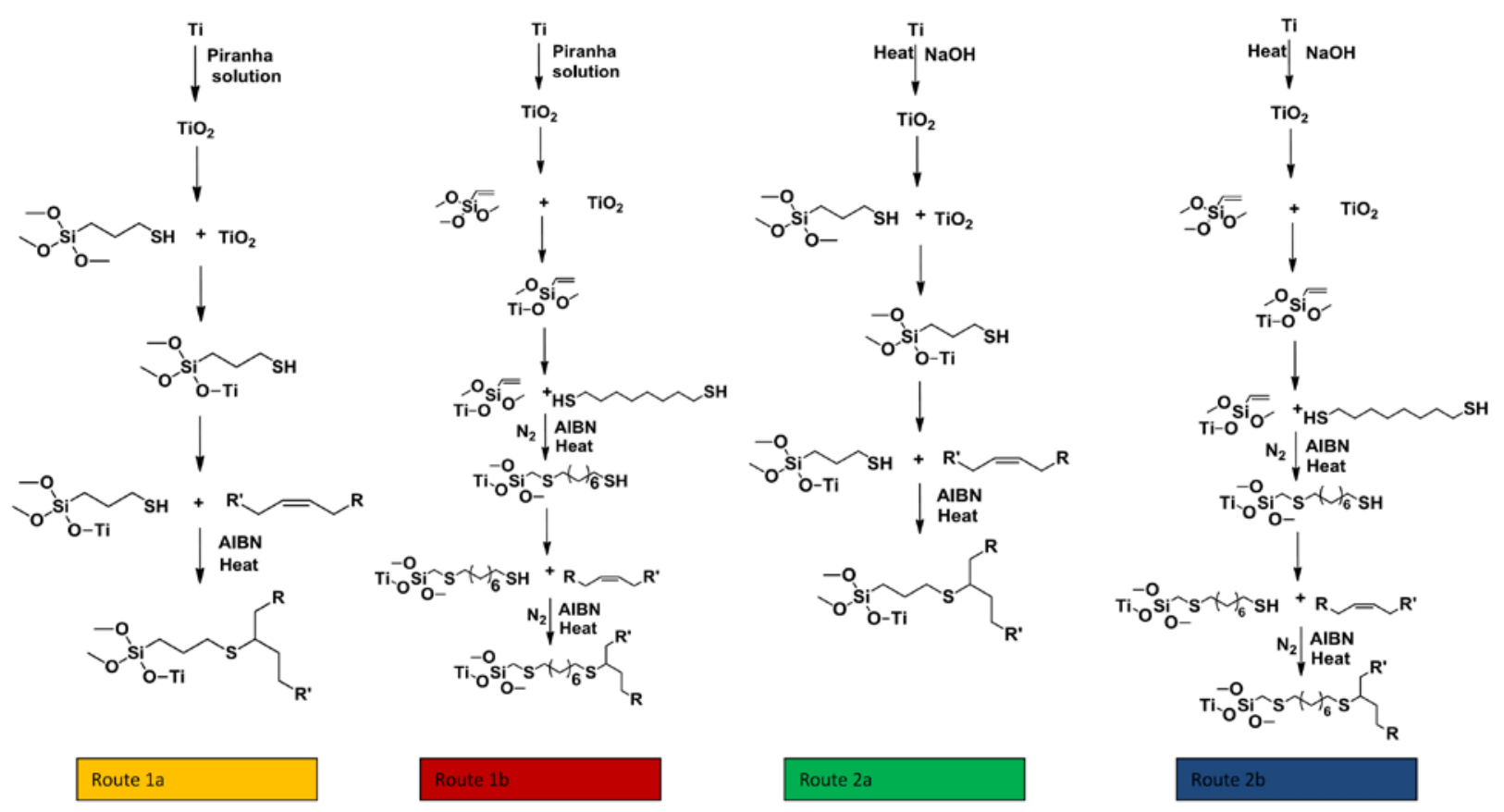

Fig. 2. Details of the different mechanisms resulting in the covalent modification of titanium with LPA. In each case the use of the thermal initiator, azobisisobutyronitrile (AIBN), enabled LPA attachment to the metal surface.

Piranha solution was prepared by combining equal volumes $(20 \mathrm{~mL})$ of ice-cold concentrated sulphuric acid and 30 $\%(\mathrm{w} / \mathrm{v})$ hydrogen peroxide [Please note that Piranha solution is highly corrosive and oxidative. Correct safety procedures should be followed at all times. Resultant solutions should not be stored, but immediately used or disposed of in a correct and safe manner]. Once mixed ( $\sim 10 \mathrm{~min})$ the resultant solution was allowed to reach room temperature before use. The covalent attachment of either LPA or FHBP involved a multi-step process. For the purposes of clarification, a summary of the different steps taken to functionalise Ti with LPA is presented (Fig. 1). Fig. 2 details the actual chemistry pertaining to Ti functionalisation by the four different routes tested.

In Route $1, \mathrm{Ti}$ samples were treated with piranha solution under gentle stirring for $2 \mathrm{~h}$ before being washed extensively with deionised water, followed by drying in desiccators. Route 1a involved the addition of a linker molecule, (3-mercaptopropyl) trimethoxysilane (MPTMS, $1 \mathrm{~mL}$ ), to batches of 8 discs immersed in $50 \mathrm{~mL}$ anhydrous toluene. Attachment of the linker took place over $24 \mathrm{~h}$ after which the discs were recovered, rinsed in toluene and dried prior to storage in desiccators. An alternative to MPTMS was also explored; in this instance (Route 1b), two reagents were used: the first step of the functionalisation process involved the addition of vinyl trimethoxysilane (VTMS, $1 \mathrm{~mL}$ ) to Ti discs in anhydrous toluene (50 $\mathrm{mL}$ ) and reacted with the discs over a $24 \mathrm{~h}$ period. For the second step, the discs were recovered, rinsed in dry toluene before re-immersion in the same solvent $(50 \mathrm{~mL})$ supplemented with 1,8 octanedithiol ( $1 \mathrm{~mL})$, followed by the radical initiator, azobisisobutyronitrile (AIBN, 25 $\mathrm{mg}$ ). Discs were heated to $60^{\circ} \mathrm{C}$ and left overnight. After the treatment period the discs were recovered, rinsed in toluene, dried in air and stored in desiccators. In the final step for both routes, $100 \mu \mathrm{L}$ of the LPA/FHBP stock was added to 8 prepared Ti samples in dry toluene $(50 \mathrm{~mL})$. Azobisisobutyronitrile (AIBN, $25 \mathrm{mg}$ ), was added and the resultant mixture heated to $60^{\circ} \mathrm{C}$ and left over night. In this regard the influence of direct versus indirect attachment of LPA/FHBP to Ti on the osteoblast response to D3 could be explored. The functionalised Ti discs were recovered, given a triple rinse in toluene and dried prior to physicochemical characterisation and human osteoblast exposure. A second method for surface functionalisation for lipid attachment to Ti was also examined (Route 2). In this particular instance untreated discs were placed in hot $\left(60^{\circ} \mathrm{C}\right)$ concentrated $\mathrm{NaOH}(10 \mathrm{M})$ and then subsequently treated in a similar manner as Route 1. 
A

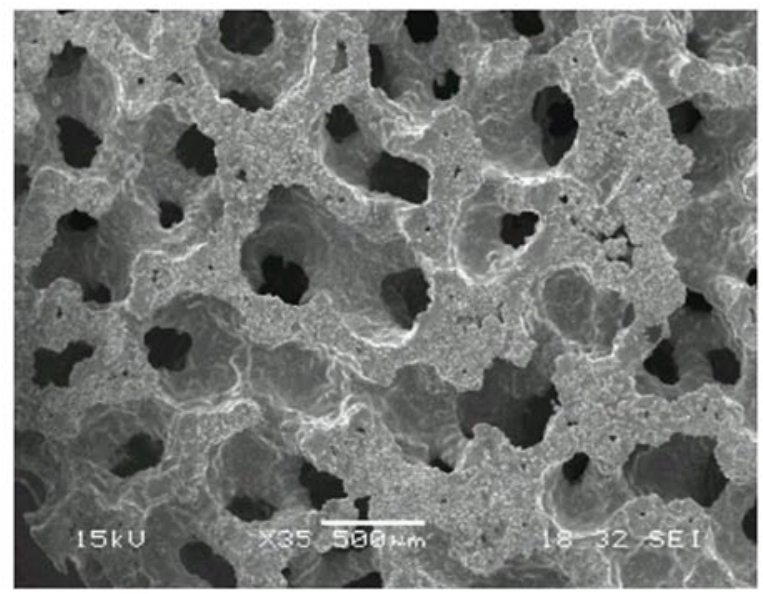

C

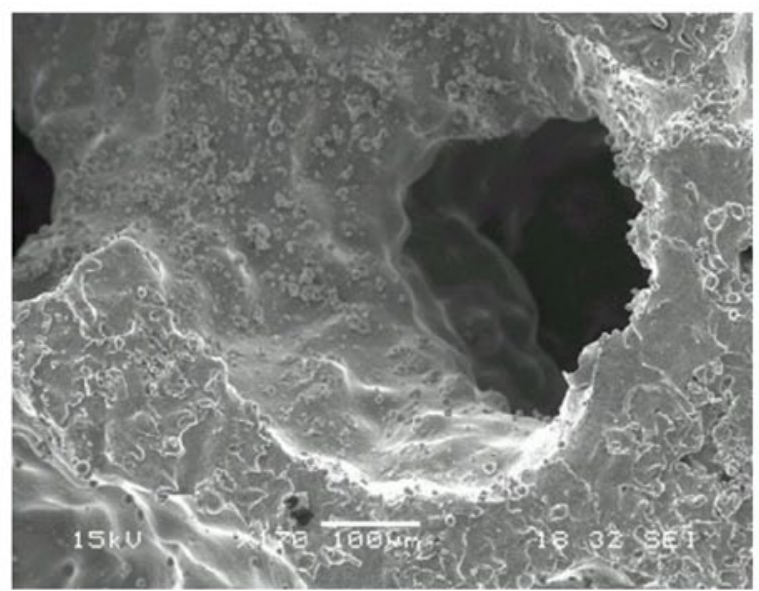

B

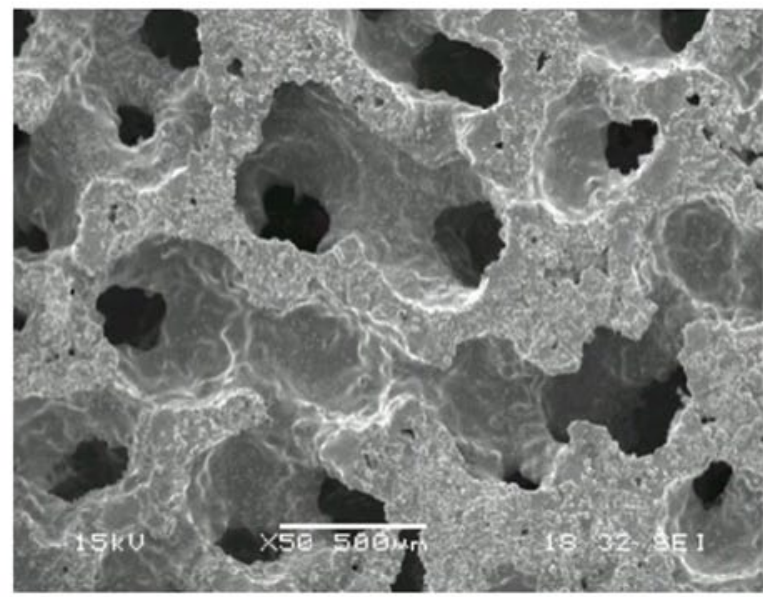

D

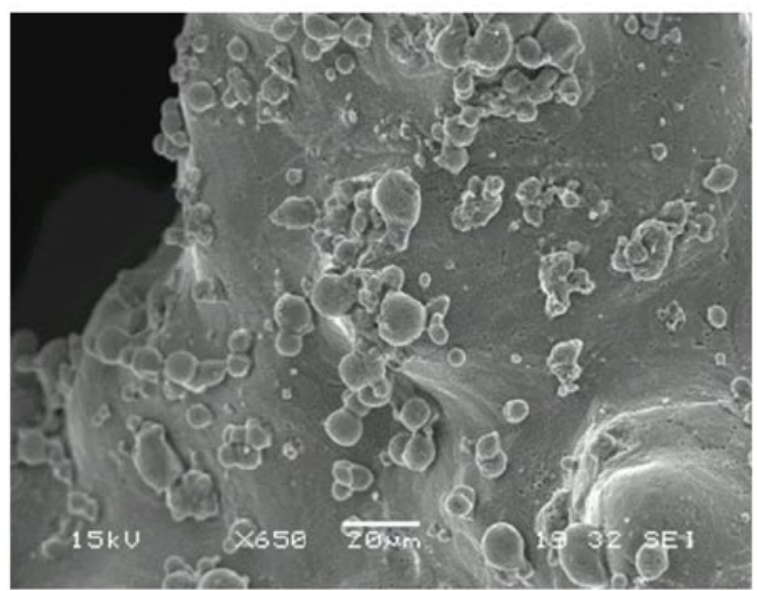

Fig. 3. Scanning electron micrographs of open porous Ti foam. A. Low magnification (original microscope magnification, $35 \mathrm{x}$ ) of a typical specimen (scale bar $500 \mu \mathrm{m}$ ). B. Original microscope magnification $50 \mathrm{x}$, depicting the clear porosity of the open structure (scale bar $500 \mu \mathrm{m}$ ). C. Ti foam as viewed at an original microscope magnification of $170 \mathrm{x}$ (scale bar $100 \mu \mathrm{m}$ ). D. Closer inspection close to one of the channels of the porous Ti sample (original microscope magnification, $650 \mathrm{x}$, scale bar $20 \mu \mathrm{m}$ ).

In each instance processed discs were stored at ambient temperature, in the dark, in glass tubes fitted with lids for one month prior to physiochemical characterisation and in vitro evaluation.

\section{Physicochemical characterisation of lipid- functionalised $\mathbf{T i}$}

Titanium foams were processed for SEM (Fig. 3) as we have described previously for solid metal (Mansell et $a l ., 2010)$. Briefly, discs were coated with a $15 \mathrm{~nm}$ layer of $\mathrm{Pt} / \mathrm{Pd}$ prior to analysis using an accelerating voltage of $10 \mathrm{kV}$ and images taken with a JEOL 6330 FEGSEM. To test for successful lipid modification of Ti, the samples (the top 1-2 atomic layers) were examined by Time-of-Flight (ToF) secondary ion mass spectrometry (SIMS) and spectra recorded using a IX23LS ToF SIMS instrument (VG Scienta Ltd, St Leonards-on-Sea, UK) with a focused gallium ion gun and Poschenreider energy analyser. SIMS analyses were performed on the samples under vacuum $\left(5 \times 10^{-9} \mathrm{mBar}\right)$ with a $1 \mathrm{nA}$ current gallium ion beam (20 keV energy) with a pulse length of $30 \mathrm{~ns}$ and $10 \mathrm{kHz}$ repetition rate. For acquisition, 1,600,000 pulses per spectrum over a $0.3 \mathrm{~mm}$ square area were obtained for each sample.

A Thermo Fisher Scientific (East Grinstead, UK) Escascope equipped with a dual anode X-ray source $\left(\mathrm{AlK}_{\alpha} 1486.6 \mathrm{eV}\right.$ and $\mathrm{MgK}_{\alpha} 1253.6 \mathrm{eV}$ ) was used for XPS analysis. Solid samples were analysed under high vacuum $\left(<5 \times 10^{-8}\right.$ mbar $)$ with $\mathrm{AlK}_{\alpha}$ radiation at $270 \mathrm{~W}(13.5 \mathrm{kV}$; $20 \mathrm{~mA}$ ). Following the acquisition of survey spectra over a wide binding energy range, the $\mathrm{C} 1 \mathrm{~s}, \mathrm{~N} 1 \mathrm{~s}, \mathrm{O} 1 \mathrm{~s}, \mathrm{P} 2 \mathrm{p}$, $\mathrm{S} 2 \mathrm{p}, \mathrm{A} 12 \mathrm{p}, \mathrm{Si} 2 \mathrm{p}, \mathrm{V} 2 \mathrm{p}, \mathrm{Ti} 2 \mathrm{p}$ spectral regions were then scanned at a higher energy resolution such that valence state determinations could be made for each element. Highresolution scans were acquired using $30 \mathrm{eV}$ pass energy and $200 \mathrm{~ms}$ dwell times. Data analysis was carried out 
using Pisces software (Dayta Systems, Bristol, UK) with binding energy values of the recorded lines referenced to the adventitious hydrocarbon $\mathrm{C} 1 \mathrm{~s}$ peak at $284.8 \mathrm{eV}$.

Contact angles were obtained for solid samples using a Krüss Drop Shape Analysis system (DSA 10 MK2) with Drop Shape Analysis software for Windows, version 1.90.0.14 calibrated to sessile drop measurements. This method does not allow for hysteresis measurements. The contact angle was an average of both right and left contact angles for each measurement. Contact angles were measured using water (between 5-7 $\mu \mathrm{L}$ ) with three measurements taken from the surface of each sample (4 samples for each of the different surface finishes). Although humidity was not recorded, experiments were carried out in a dedicated and contained air-conditioned laboratory.

\section{Application of human osteoblasts to lipid- functionalised Ti}

Human osteoblast-like cells (MG63) were cultured in conventional tissue culture flasks $(250 \mathrm{~mL}$, Greiner, Frickenhausen, Germany) in a humidified atmosphere at 37 ${ }^{\circ} \mathrm{C}$ and $5 \% \mathrm{CO}_{2}$. Although osteosarcoma-derived, MG63 cells exhibit features in common with human osteoblast precursors or poorly differentiated osteoblasts. Specifically, these cells produce type I collagen with no or low basal osteocalcin (OC) and alkaline phosphatase (ALP). However, when MG63s are treated with D3, both OC and ALP increase which are features of the osteoblast phenotype (Clover and Gowen, 1994). Consequently, the application of these cells to assess the potential pro-maturation effects of novel materials is entirely appropriate. Further, the MG63 continues to be the most widely used osteoblast-like cell line in biomaterials research (Bachle and Kohal, 2004). Cells were grown to confluence in Dulbecco's modified Eagle medium (DMEM)/F12 nutrient mix (Gibco, Paisley, Scotland) supplemented with sodium pyruvate ( $1 \mathrm{mM}$ final concentration), L-glutamine (4 mM), streptomycin (100 $\mathrm{ng} / \mathrm{mL})$, penicillin $(0.1$ units $/ \mathrm{mL})$ and $10 \% \mathrm{v} / \mathrm{v}$ foetal calf serum (Gibco, Paisley, Scotland). The growth media (500 $\mathrm{mL}$ final volume) was also supplemented with $5 \mathrm{~mL}$ of a 100x stock of non-essential amino acids. Once confluent, MG63s were subsequently dispensed into blank 24-well plates (Greiner, Frickenhausen, Germany) or plates containing either control (piranha/ $\mathrm{NaOH}$ treated) or LPA/ FHBP-modified titanium discs. In each case, wells were seeded with $1 \mathrm{~mL}$ of a $4 \times 10^{4}$ cells $/ \mathrm{mL}$ suspension (as assessed by haemocytometry) spiked with D3 to a final concentration of $100 \mathrm{nM}$. For the porous Ti samples, cells were seeded in the same growth medium but devoid of phenol red to eliminate any interference with the ALP assay described below. Cells were then cultured for $3 \mathrm{~d}$, the media removed and the cells processed for total ALP activity to ascertain the extent of cellular maturation. Having identified which of the functionalised Ti substrates was the best at supporting D3-induced osteoblast maturation, all subsequent studies focussed on this particular surface modification (Route 1a).

In a separate study, the potential of recycling the lipidfunctionalised discs was explored to ascertain whether the modified metal was still able to support an osteoblast maturation response. Briefly, discs that had already been seeded with cells and processed for total ALP activity were recovered, rinsed under running tap water and scrubbed with a toothbrush to remove any remaining cellular debris. Once rinsed, the samples were immersed in $70 \%$ aqueous ethanol and left for 2-3 min before being rinsed several times with sterile PBS. Washed discs were then placed into clean, multi-well plates, rinsed with DMEM/ F12 nutrient mix and subsequently seeded with MG63 cells as described above. A second repeat of this step was performed to explore how three independent uses of the same covalently functionalised discs could support the maturation of MG63 osteoblasts to D3.

\section{Cell number}

An assessment of cell number was performed using a combination of the tetrazolium compound 3-(4,5-dimethylthiazol-2-yl)-5-(3-carboxymethoxyphenyl)-2-(4-sulfophenyl)-2H-tetrazolium, innersalt (MTS, Promega, UK) and the electron-coupling reagent phenazine methosulphate (PMS). Each compound was prepared separately in pre-warmed $\left(37^{\circ} \mathrm{C}\right)$ phenol red-free $\mathrm{DMEM} / \mathrm{F} 12$, allowed to dissolve, and then combined so that $1 \mathrm{~mL}$ of a $1 \mathrm{mg} / \mathrm{mL}$ solution of PMS was combined to $19 \mathrm{~mL}$ of a $2 \mathrm{mg} / \mathrm{mL}$ solution of MTS. A stock suspension of MG63s ( $1 \times 10^{6}$ cells $\left./ \mathrm{mL}\right)$ was serially diluted in growth medium to give a series of known cell concentrations down to $25 \times 10^{3}$ cells $/ \mathrm{mL}$. Each sample ( $0.5 \mathrm{~mL}$ in a microcentrifuge tube) was spiked with 0.1 $\mathrm{mL}$ of the MTS/PMS reagent mixture and left for 45 min within a tissue culture cabinet. Once incubated, the samples were centrifuged at $900 \mathrm{rpm}$ to pellet the cells and $0.1 \mathrm{~mL}$ of the supernatants dispensed onto a 96-well microtitre plate and the absorbances at $492 \mathrm{~nm}$ read using a multiplate reader. Plotting the absorbances against known cell number, as assessed initially using haemocytometry, enabled extrapolation of cell numbers for the experiments described herein.

\section{Total ALP activity}

An assessment of ALP activity is reliably measured by the generation of $p$-nitrophenol ( $p-N P)$ from p-nitrophenylphosphate ( $\mathrm{p}-\mathrm{NPP}$ ) under alkaline conditions. The treatment of cells to quantify ALP activity was similar to that described by us recently (Mansell et al., 2010). Briefly, the MTS/PMS reagent was removed and the monolayers incubated for a further $15 \mathrm{~min}$ in fresh phenol red-free DMEM/ F12 to remove the residual formazan. Following this incubation period, the medium was removed and the monolayers lysed with $0.1 \mathrm{~mL}$ of $25 \mathrm{mM}$ sodium carbonate ( $\mathrm{pH} 10.3), 0.1 \%$ (v/v) Triton X-100. After 2 min, each well was treated with $0.2 \mathrm{~mL}$ of $15 \mathrm{mM} \mathrm{p}-\mathrm{NPP}$ (di-Tris salt, Sigma, UK) in $250 \mathrm{mM}$ sodium carbonate ( $\mathrm{pH}$ 10.3), 1 $\mathrm{mM} \mathrm{MgCl}$. Lysates were then left under conventional cell culturing conditions for $1 \mathrm{~h}$. After the incubation period, 0.1 $\mathrm{mL}$ aliquots were transferred to 96-well microtitre plates and the absorbance read at $405 \mathrm{~nm}$. An ascending series of p-NP (25-500 $\mu \mathrm{M})$ prepared in the incubation buffer enabled quantification of product formation. To ensure that the ALP activity was only associated for cells attached to $\mathrm{Ti}$ and not to the surrounding plastic, the sample discs were transferred to clean multiwell plates and then processed for 
Fig. 4. The LPA3 selective agonist, FHBP, synergistically co-operates with D3 to promote human osteoblast maturation. The ability of selected agents to promote the formation of mature osteoblasts can reliably be assessed through determining cellular alkaline phosphatase (ALP) activity via the production of p-nitrophenol (p-NP). The data depicted clearly highlight the ability of FHBP (250 nM - $2000 \mathrm{nM})$ to synergistically co-operate with D3 (100 $\mathrm{nM}$ ) in a time and dose-dependent fashion.

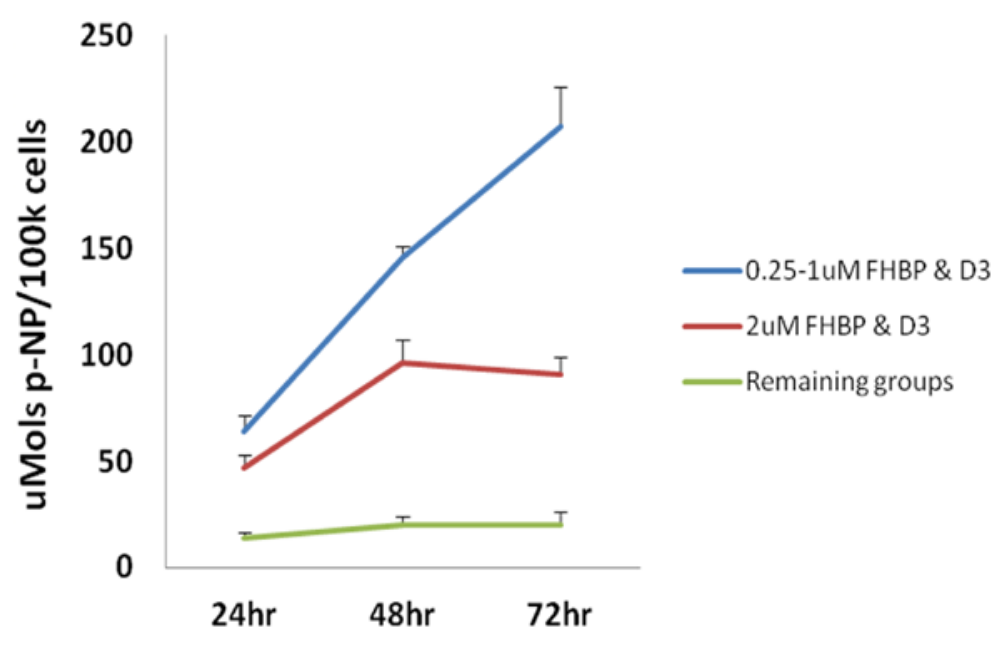

Table 2. Total ALP activity for MG63 cells treated with D3 and the LPA3 selective agonist, FHBP.

\begin{tabular}{lccc} 
Time point $^{\mathbf{1}}$ & $\mathbf{2 4} \mathbf{~ h}$ & $\mathbf{4 8} \mathbf{~ h}$ & $\mathbf{7 2} \mathbf{~ h}$ \\
\hline Vehicle & $12.7 \pm 0.9$ & $13.1 \pm 1.1$ & $10.1 \pm 0.6$ \\
D3 only & $19.0 \pm 1.2$ & $18.7 \pm 1.4$ & $16.8 \pm 1.1$ \\
$0.25 \mu \mathrm{M}$ FHBP & $13.8 \pm 0.8$ & $24.0 \pm 1.4$ & $27.3 \pm 1.8$ \\
$0.5 \mu \mathrm{M}$ FHBP & $14.2 \pm 1.2$ & $23.6 \pm 2.1$ & $23.7 \pm 1.5$ \\
$1.0 \mu \mathrm{M}$ FHBP & $12.1 \pm 0.7$ & $22.7 \pm 1.3$ & $24.7 \pm 1.7$ \\
$2.0 \mu \mathrm{M}$ FHBP & $12.5 \pm 1.1$ & $15.8 \pm 1.2$ & $15.6 \pm 0.9$ \\
$0.25 \mu \mathrm{M}$ FHBP \& D3 & $71.9 \pm 4.7$ & $151.0 \pm 8.9$ & $225.5 \pm 21.7$ \\
$0.5 \mu \mathrm{M}$ FHBP \& D3 & $62.4 \pm 5.3$ & $145.6 \pm 16.1$ & $207.4 \pm 18.5$ \\
\hline
\end{tabular}

${ }^{1}$ The data are graphically represented in Fig. 4.

ALP activity. Unless stated otherwise, total ALP activity is expressed as the mean micromolar concentration of $\mathrm{p}-\mathrm{NP}$ per 100,000 cells, as extrapolated from the MTS/PMS assay described above.

\section{Statistical analysis}

Unless stated otherwise, all the cell culture experiments described above were performed three times and all data were subject to a one-way analysis of variance (ANOVA) to test for statistical significance as we have reported previously (Mansell et al., 2010). When a $p$ value of $<$ 0.05 was found, a Tukey multiple comparisons post-test was performed between all groups. All data are expressed as the mean together with the standard deviation.

\section{Results}

The novel LPA3 selective agonist, FHBP, synergistically co-operates with D3 to enhance MG63 maturation

Time (24, 48 and $72 \mathrm{~h})$ and dose (250-2000 $\mathrm{nM})$ response studies were conducted for FHBP to examine its ability to evoke a maturation response in human osteoblasts, either alone or in combination with $100 \mathrm{nM} \mathrm{D}$. The findings presented clearly indicate that FHBP acts synergistically with D3 to promote statistically significant time and dosedependent increases in p-NP and therefore ALP activity in
MG63 cells (Fig. 4). The findings of demonstrably elevated levels of ALP were independent of cell number (Table 2).

\section{Physiochemical evidence for the successful covalent conjugation of LPA to titanium}

Time-of-Flight Secondary Ion Mass Spectrometry (ToF SIMS) was used to determine the presence of LPA on the prepared modified titanium. The LPA phosphate head group produced characteristic peaks at 63 and $79 \mathrm{~m} / \mathrm{z}$ corresponding to $\mathrm{PO}_{2}^{-}$and $\mathrm{PO}_{3}^{-}$, respectively. These signals were recorded from the negative ion spectrum of the functionalised discs, indicating the presence of phosphatecontaining LPA on the metal surface. These characteristic $\mathrm{PO}_{2}^{-}$and $\mathrm{PO}_{3}^{-}$signals were absent for non-functionalised control discs (Fig. 5).

In order to provide further evidence of surface functionalisation, X-ray photoelectron spectroscopy (XPS) was used to analyse the surfaces of both functionalised and control samples (Table 3). The piranha-treated control samples yielded carbon (37.7\%), oxygen (35.8\%) and sulphur $(11.3 \%)$, whereas the silane-functionalised samples exhibited carbon (49.2\%), oxygen (24.3\%), silicon $(17.3 \%)$ and sulphur $(2 \%)$. It is noteworthy that no phosphorus was detected on either of the controls. The large carbon content for the piranha sample is tentatively attributed to adsorption after drying. The presence of oxygen and sulphur on the piranha-treated control sample are a consequence of the treatment in the strongly 


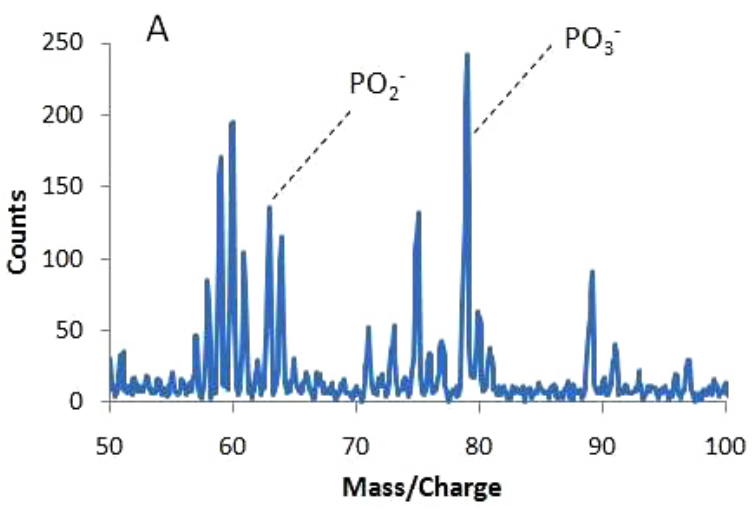

Fig. 5. Physiochemical evidence for the successful covalent conjugation of LPA to titanium. Time of Flight Secondary Ion Mass Spectrometry (ToF SIMS) was used to determine the presence of LPA on modified titanium. A. The LPA phosphate head group produced characteristic peaks at 63 and $79 \mathrm{~m} / \mathrm{z}$ corresponding to $\mathrm{PO}_{2}^{-}$and $\mathrm{PO}_{3}^{-}$, respectively. These signals were recorded from the negative ion spectrum of the functionalised discs indicating the presence of phosphate-containing LPA on the metal surface. $\mathbf{B}$. The characteristic $\mathrm{PO}_{2}{ }^{-}$and $\mathrm{PO}_{3}^{-}$- signals were absent from the same spectrum for non-functionalised control discs.

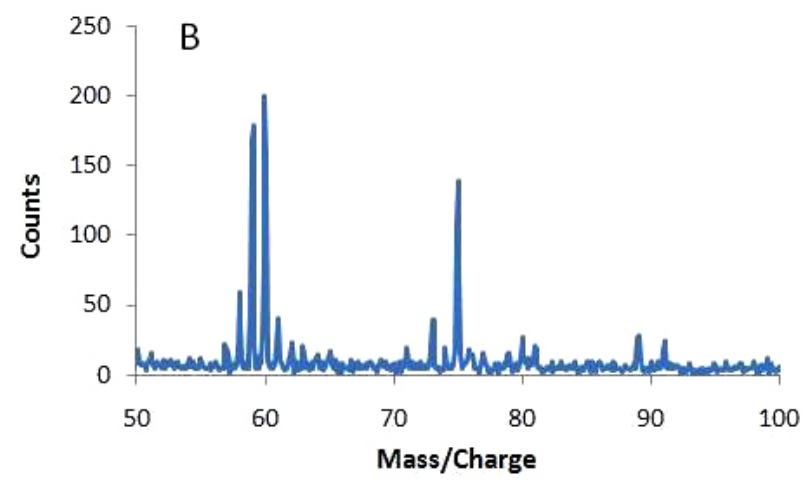

Table 3. X-ray photoelectron spectroscopy (XPS) data obtained for each of the three different surface treatments of solid titanium.

\begin{tabular}{lccc} 
Element & Piranha & $\begin{array}{c}\text { Silane } \\
\text { Atomic } \%\end{array}$ & Functionalised \\
\hline Carbon 1s & 37.7 & 49.2 & 50.9 \\
Nitrogen 1s & 4.6 & 0.4 & 0.8 \\
Oxygen 1s & 35.8 & 24.3 & 24.0 \\
Aluminium 2p & 1.1 & 0.9 & 1.5 \\
Silicon 2p & - & 17.3 & 11.6 \\
Potassium 2p3 & 1.0 & - & - \\
Phosphorous 2s & - & - & 0.8 \\
Sulphur 2p3 & 11.3 & 2.0 & 5.2 \\
\hline
\end{tabular}

oxidising sulphur-containing piranha solution. This not only hydroxylates the surface but also appears to deposit sulphate ions onto it. However, there is no indication of either phosphorus or silicon on the piranha-treated control. The increase in carbon content and the presence of silicon were expected, and attributed to the addition of the silane coupling agent.

Analysis of the LPA-functionalised sample showed the presence of silicon $(11.6 \%)$, phosphorus $(0.8 \%)$ as well as an increase in carbon content $(50.9 \%)$. Theoretical calculations, based on the molecular volumes of the species involved, were performed to quantify whether the percentage of phosphorus (from LPA) was consistent with good surface coverage. Using these calculations we estimated that our covalent approach yielded coverage of $45.8 \%$ of the maximum theoretical coverage. This finding, as determined from the XPS data, was an outcome of the process utilised. However, we were unable to increase surface coverage when using more LPA and/or extending the incubation time (data not shown).

Contact angle measurements (Table 4) were used to determine the influence of surface groups (i.e., whether the surface of the samples was hydrophobic or hydrophilic), and to provide further indirect proof whether successful attachment of the LPA had occurred. Four samples were measured; a control (untreated) sample, a piranhatreated sample, a silane-treated specimen and an LPA functionalised sample. Both the piranha and untreated control samples yielded hydrophilic surfaces, with average contact angles of $35.5^{\circ}$ and $67^{\circ}$ respectively. The greater hydrophilicity of the piranha sample is attributed to the high degree of hydroxylation of the sample surface. An increase in contact angle was observed for the silane treated sample with an average angle of $119.7^{\circ}$. As attached LPA would present a hydrophilic head group it would be expected that the contact angle would decrease from that of the 
Control

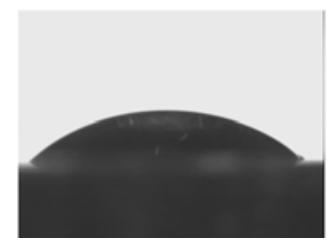

Piranha

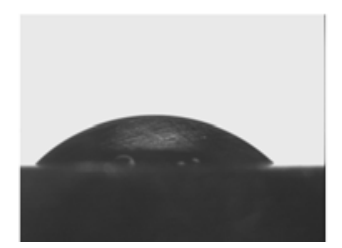

Silane

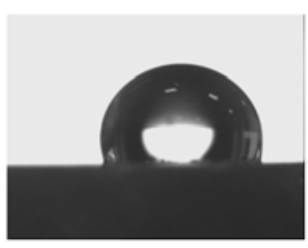

Silane plus LPA

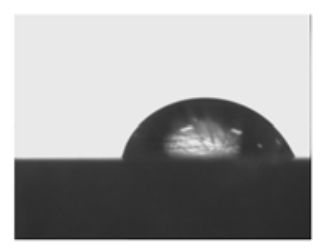

Fig. 6. Typical morphologies of the water drops as visualised for the different titanium surface treatments.

Table 4. A summary of the contact angle data obtained from four different solid titanium samples.

\begin{tabular}{lcccc} 
Sample $^{1}$ & Angle 1 $^{\circ}$ & Angle 2 $^{\circ}$ & Angle 3 $^{\circ}$ & Average $^{\circ}$ \\
\hline Control & 62.5 & 67.2 & 71.2 & 67.0 \\
Piranha & 34.7 & 35.7 & 36.0 & 35.5 \\
Silane & 122.0 & 117.6 & 119.4 & 119.7 \\
Silane plus LPA & 76.0 & 85.0 & 78.0 & 79.7 \\
\hline
\end{tabular}

${ }^{1}$ Contact angles were measured using water with three measurements taken from the surface of each sample. For the sake of clarity the corresponding images depicting the droplet shapes for each of the different surfaces are provided (Fig 6).

silane sample. This trend was indeed observed, with the contact angle decreasing to an average of $79.7^{\circ}$, showing that LPA had been successfully attached to the sample. Fig. 6 depicts the morphologies of the water drops for the different surface treatments.

These clear changes in surface polarity are reflected in our findings for early attachment $(2 \mathrm{~h})$ and spreading $(24 \mathrm{~h})$ of MG63 cells upon solid Ti discs. The findings presented indicate persistence of more rounded morphologies for cells seeded on to LPA-functionalised surfaces compared to the piranha controls, even after $24 \mathrm{~h}$ (Fig. 7).

Lipid-functionalised solid $\mathrm{Ti}$ is a better substrate for supporting D3-induced osteoblast maturation than unmodified metal

Initially, we screened solid Ti discs that had been subject to the described LPA functionalisation treatment regimens to ascertain which route yielded the most robust maturation response for MG63 cells. The data (Fig. 8) indicate that Route 1a (i.e., the LPA-functionalised discs exposed to initial piranha solution treatment followed by the direct attachment of the lipid) produced a modified surface with the greatest $\left({ }^{\mathrm{a}} \mathrm{p}<0.002\right.$ compared to the piranha control) potential to promote D3-induced osteoblast maturation, as supported by the greater total ALP activity. From this point on all subsequent modifications of Ti adopted this strategy of functionalisation. In a second series of experiments (Fig. 9) Ti discs were processed using Route 1a for attachment of either LPA or the LPA1/3 selective agonist, FHBP, and seeded with MG63 cells in the presence of $100 \mathrm{nM}$ D3. After $72 \mathrm{~h}$ of culture the discs were treated to assess total ALP activity. The data clearly indicate that "first use" discs coated with either LPA $\left({ }^{\mathrm{a}} p<0.001\right)$ or FHBP $\left({ }^{\mathrm{b}} p<0.001\right)$ support the maturation of human osteoblasts compared to control discs. The discs were then recovered from the wells, treated and subsequently re-seeded with cells to ascertain whether these recycled discs still exhibited bioactivity. Although total ALP activity was less for cells associated with "second use" discs, the data do support that covalent attachment of LPA is effective, and recycled samples do exhibit greater capacity to support human osteoblast maturation compared to controls. Similar results were obtained for "third use" discs, with the maturation response of cells to FHBP coating being superior ( ${ }^{c} p<0.001$ ) to that of LPA. Collectively, the data support the robust covalent attachment of biologically active LPA receptor agonists to orthopaedic grade solid titanium.

\section{Evidence for the successful LPA/FHBP-} functionalisation of open, porous Ti foam Both LPA and the selective LPA3 receptor agonist, FHBP, were immobilised onto porous Ti using Route 1a (i.e., initial piranha solution treatment followed by the direct attachment of the lysophospholipids to the metal surface). The data depicted for total ALP activity provide a clear indication that both LPA and FHBP-modified Ti foams serve as superior substrates for D3-induced MG63 maturation compared to unmodified metal $\left({ }^{\mathrm{a}} p<0.001\right.$, Fig. 10A). Evidence for osteoblast adhesion to the Ti foam is also presented (Fig. 10B) as a scanning electron micrograph image.

\section{Discussion}

Surface functionalisation of titanium with bioactive molecules is a key feature of contemporary biomaterials research, indeed a concerted global effort has ensued to enhance the performance and longevity of titanium through surface modifications that include both non-covalent and covalent functionalisation strategies (Beutner et al., 2010 and references therein). With regard to biological agents, 


\section{Ctrl-Ti 2 h}

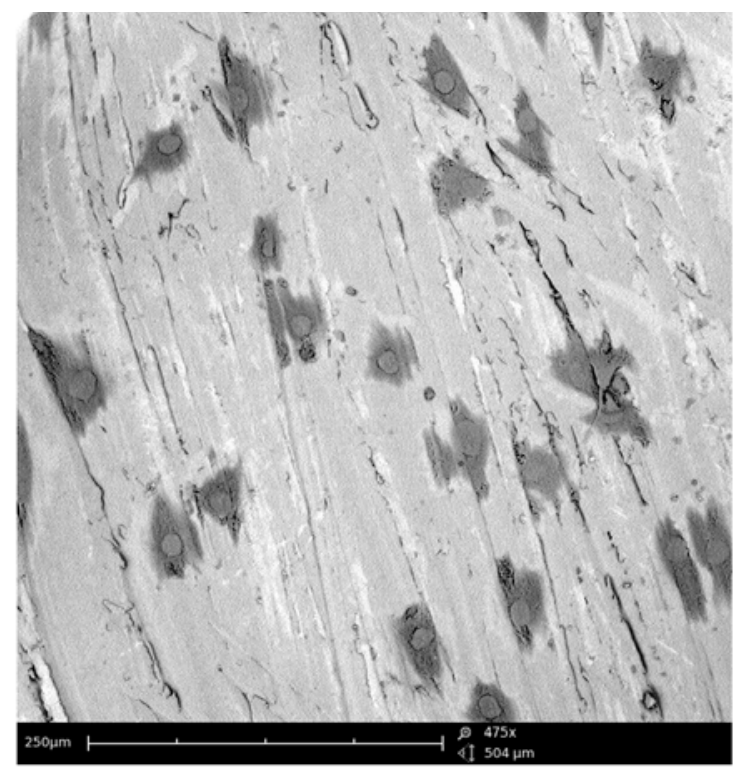

\section{LPA-Ti 2 h}

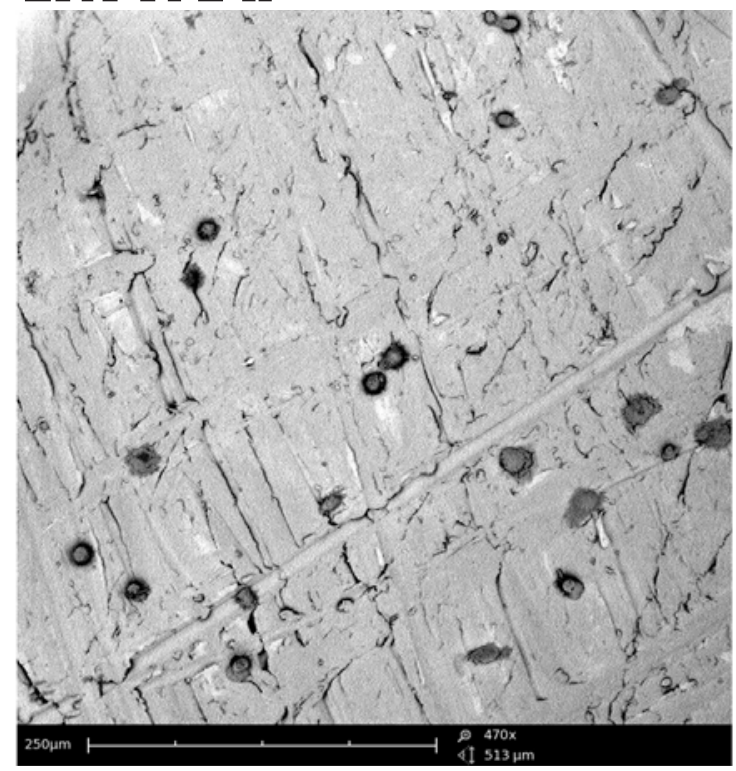

\section{$24 \mathrm{~h}$}

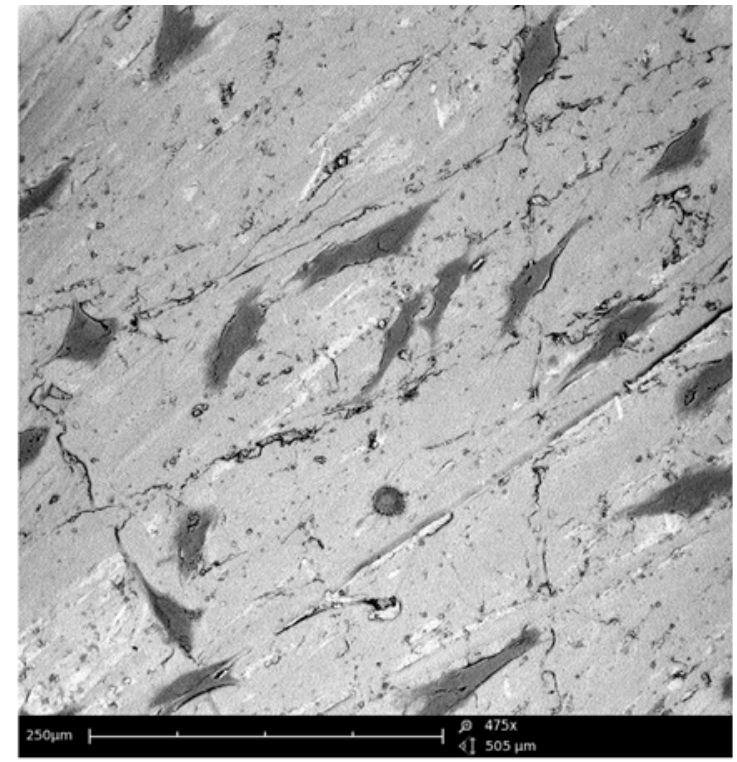

\section{$24 \mathrm{~h}$}

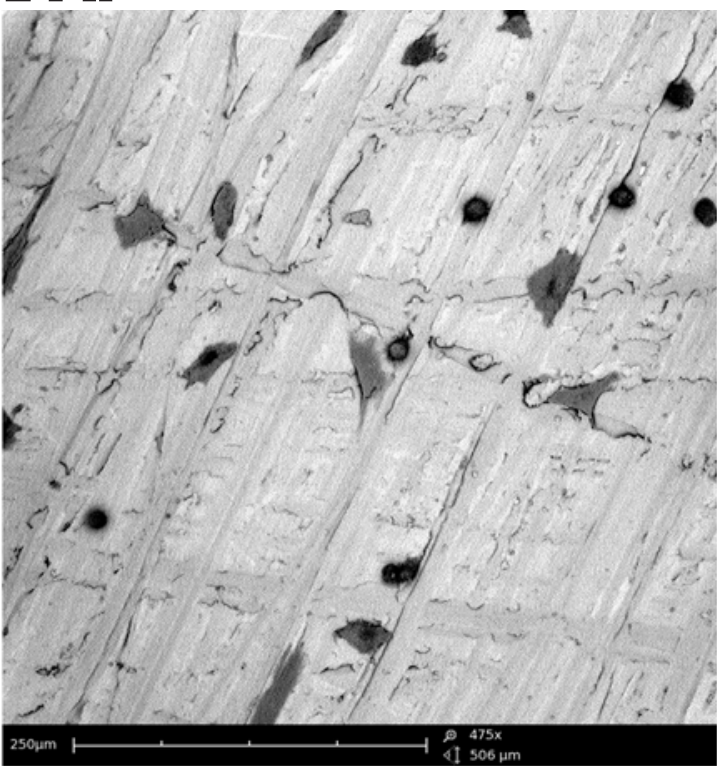

Fig. 7. Cell morphologies of MG63 osteoblasts seeded onto LPA-functionalised solid titanium. Surface modifications of titanium (Ti) can often yield alterations in substrate polarity which in turn can influence the early attachment $(2$ h) and subsequent spreading $(24 \mathrm{~h})$ of cells. The SEM images depicted (original microscope magnification $475 \mathrm{x}$, scale bar $250 \mu \mathrm{m}$ ) reveal many more rounded MG63 cells associated with the LPA-functionalised Ti compared to the piranha-treated control discs.

the molecules chosen are invariably proteins/peptides that either influence osteoblast adhesion (typically molecules that bind to cell surface integrins), or they are growth factors known to drive osteogenesis. With reference to cell adhesion molecules, researchers have examined the potential of Arg-Gly-Asp (RGD)-containing peptide coatings to aid both osteoblast attachment and to mimic the signalling incurred via the collagenous matrix they would ordinarily secrete. However, the in vivo evidence indicates that these material modifications offer, at best, modest improvements on the process of osseointegration (Elmengaard et al., 2005a; Elmengaard et al., 2005b;
Kantlehner et al., 2000; Lutz et al., 2010; Schliephake et al., 2002). Suffice it to say, bone biomaterials modified this way are not being used as functional adjuncts for bone repair and/or regeneration.

The focus for the use of growth factors primarily pertains to non-covalent interactions of large polypeptides/ proteins with bone biomaterials, for example, transforming growth factor- $\beta$, platelet-derived growth factor, insulin-like growth factors and, most significantly, bone morphogenic proteins (BMPs). Indeed, the steps taken to explore the benefits of, for example, BMPs in a biomaterials setting have focussed on (non-covalent) adsorption techniques, 


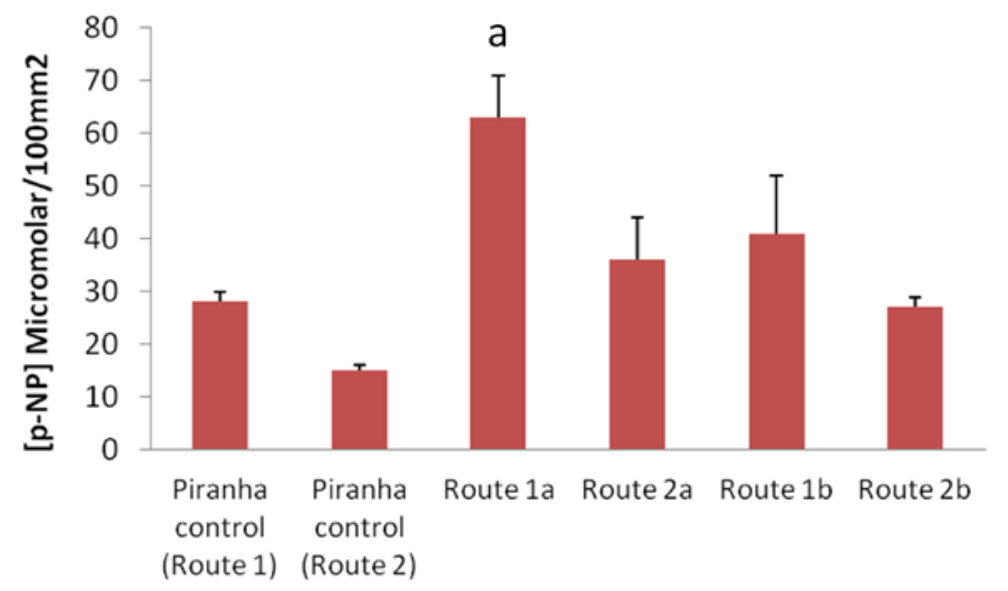

Fig. 8. Differential maturation responses of human osteoblasts to alternative LPA functionalisation steps for solid titanium. To ascertain the best approach to functionalising solid Ti discs with LPA samples where initially oxidised with piranha (Pir) solution at ambient temperature (Route 1$)$ or hot $\left(60^{\circ} \mathrm{C}\right) 10 \mathrm{M} \mathrm{NaOH}$ (Route 2). In addition the differently oxidised materials were coupled to LPA by a direct bonding method using a dithiolsilane (Route a) or via an indirect path using a vinyl silane (Route b). The data reflect total alkaline phosphatase (ALP) activity as the mean micromolar concentration of p-NP generated from $\mathrm{p}$-NPP together with the SD. The surface with the greatest potential to promote D3-induced osteoblast maturation ( ${ }^{\mathrm{a}} p<0.002$ compared to the piranha control) was generated via intial treatment with piranha followed by the direct attachment of LPA after thiosilane exposure (Route 1a). In each instance 4 replicates per treatment group were employed.

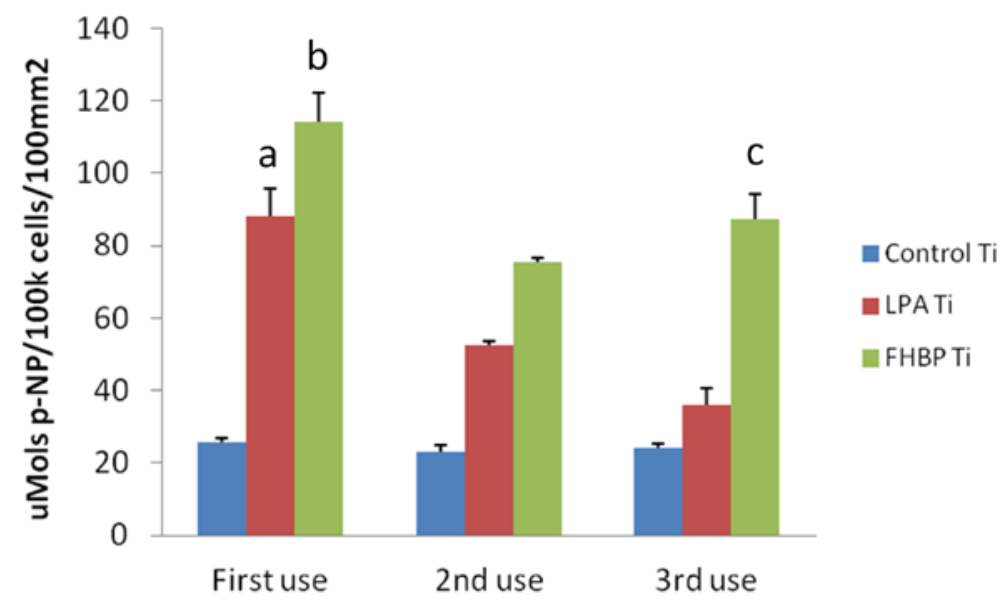

Fig. 9. Recycled lipid-functionalised solid titanium is still able to support D3-induced osteoblast maturation. Titanium discs were processed for the direct, covalent attachment of either LPA or the LPA3 selective agonist, FHBP (Route 1a). Osteoblast maturation was assessed via alkaline phosphatase (ALP) monitored by p-NP production. The data (mean $\pm \mathrm{SD}$ ) clearly indicate that "first use" discs coated with either LPA $\left({ }^{\mathrm{a}} p<0.001\right)$ or FHBP $\left({ }^{\mathrm{b}} p<0.001\right)$ support the maturation of human osteoblasts compared to the control discs. The discs were subsequently recovered from the wells, before being re-seeded with cells to ascertain whether these recycled discs still exhibited bioactivity. Although the production of p-NP is less for cells associated with "second use" discs, it is greater than controls. Similar results were obtained for "third use" discs although the maturation response of cells to FHBP coating is superior ( $p<<0.001)$ to that of LPA. Collectively the data support the robust attachment of biologically active LPA receptor agonists to orthopaedic grade solid titanium.

e.g., incorporation into the actual Ti oxide layer (Wikesjo et al., 2008), carriage within mineral phase coatings (Liu et al., 2005; Liu et al., 2007; Stenport et al., 2003; Wikesjo et al., 2002), dispersal via biodegradable scaffolds such as collagen sponges (Boyne and Jones, 2004; Tatakis et al., 2002) or delivery using type I collagen telopeptides (Bessho et al., 1999). However, the high cost of these innovative strategies using recombinant BMPs likely precludes wide scale application. Also, their large size is predicted to pose potential attachment and delivery problems at or around biomaterials as well as possible steric constraints in targeting cell surface receptors. Importantly, at the time of our study, there have been no reports describing the successful, covalent attachment of a growth factor known to influence human osteoblast formation and/or maturation at a Ti surface.

Given the potential problems posed by large biomacromolecules, we considered far smaller growth factors that might be more suitable for covalent attachment to biomaterial surfaces. The rationale behind this approach 


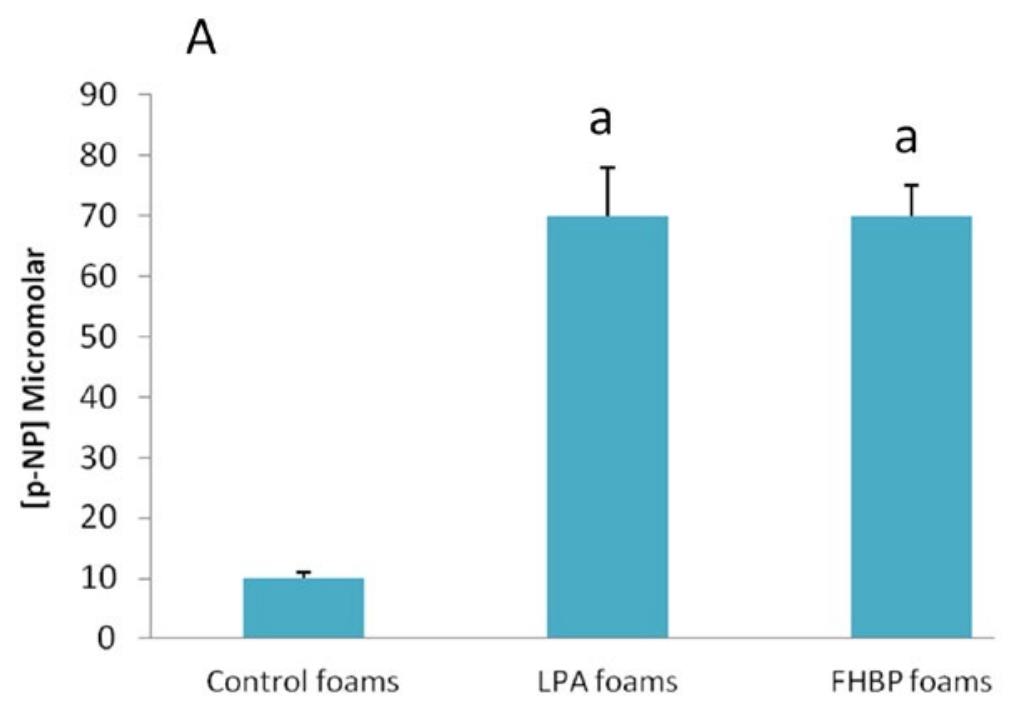

Fig. 10. Open-porous titanium foam can be lipid-functionalised to enhance human osteoblast maturation. A. Both LPA and the selective LPA3 receptor agonist, FHBP, were immobilised onto porous $\mathrm{Ti}$ using initial piranha solution treatment followed by their direct attachment to the metal surface (Route 1a). The data depicted for total ALP activity provides a clear indication that both LPA and FHBP modified Ti foams serve as superior substrates for D3-induced MG63 maturation compared to unmodified metal $\left({ }^{\mathrm{a}} p<0.001\right)$. B. Scanning electron micrograph evidence for osteoblast adhesion within one of the pores of the Ti foam. Note the clear points of cell attachment (arrows) and cell spreading across the metal surface.

B

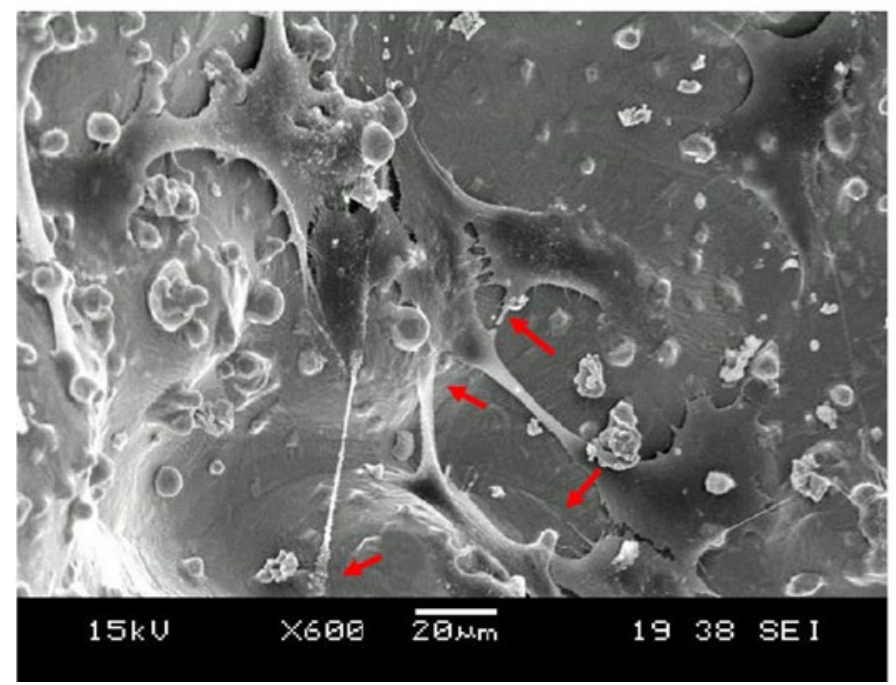

is to preserve (or even enhance) their signalling capabilities and reduce the potential of steric hindrance to their cell surface receptor proteins. One particularly attractive candidate for Ti bio-functionalisation is the small lipid LPA and certain LPA receptor agonists, e.g., (2S)-OMPT and FHBP. Of particular significance to bone formation, and therefore the potential to secure a mechanically sound mineralised matrix, is our discovery that LPA/(2S)-OMPT (and now FHBP) can synergistically co-operate with D3 to promote human osteoblast maturation at the titanium surface. A large increase in total ALP, an enzyme essential for bone collagen calcification (Whyte, 2010), was used as a reliable indicator of osteoblast maturation. It was this striking co-operative influence of D3 and LPA on cellular maturation (Gidley et al., 2006) that led us to focus on LPA and related lipids and explore routes to their covalent attachment to Ti. We restricted our evaluation of osteoblast maturation via ALP activity because of its importance to mineralisation. Although $\mathrm{OC}$ is a bone fide marker of the osteoblast phenotype there has been an emerging body of evidence implicating $\mathrm{OC}$ in whole body energy metabolism rather than participating in skeletal calcification. Recent reviews by eminent bone biologists now propose a bone-pancreas endocrine loop to help explain the biological action of $\mathrm{OC}$ in insulin sensitivity, action and energy metabolism (Clemens and Karsenty, 2011; Ducy, 2011). Suffice it to say, OC ablation does not result in a skeletal phenotype whereas a loss-offunction mutation in the TNSALP gene encoding for ALP results in hypophosphatasia, a condition characterised by inadequately mineralised bone (Whyte, 2010). Since ALP is tightly linked to bone matrix ossification, any factors promoting its expression have the potential to favour competent bone formation, including, for example, at implant surfaces.

We deliberately sought a covalent method of attachment primarily to ensure that the lipids are more likely to remain bound when subject to the forces encountered during implant insertion and physical activity post-operatively. In order to ensure that the phosphate head group of the LPA/FHBP would be accessible to cell surface receptors, we focussed our attention on the alkene moiety for 
covalent modification. The use of thiol-ene chemistry presented itself as a facile and relatively mild reaction route for the covalent fixation of the selected lipid species (Ganeva et al., 2003a; Ganeva et al., 2003b). Appropriate preparation of the Ti surface enables the use of silane-based functionalisation to present either a thiol reactive group for direct radical-mediated addition to the LPA double bond (Route 1a), or, as an alternative, functionalisation with an alkene-modified silane for further reaction with a dithiol (Route 1b). The possible drawback of the dithiol species reacting twice with surface-anchored alkene groups (and thus not being available to react with LPA in the following step) was confirmed by the lower functional activity displayed by discs modified this way. Suffice it to say, the most reliable method towards achieving a superior, bioactive Ti surface was via the direct attachment of the lysophospholipids following piranha solution surface pretreatment.

Herein, we provide clear evidence for the successful functionalisation of both solid and porous Ti with either LPA or the LPA receptor agonist, FHBP. These novel biomaterials successfully co-operated with D3 to secure human osteoblast maturation, as supported by the marked, synergistic increases in total ALP activity. Having initially seeded MG63 cells on to control and lipid-modified Ti, the samples were recovered, rinsed under running tap water, cleaned and reused for both a second and third time. The primary purpose of this series of experiments was to provide evidence that the LPA/FHBP could remain attached with recycling of the Ti samples. Although Ti prostheses are not "recycled" it is worth pointing out however that during the process of arthroplasty, any devices that do not fit at first attempt are recovered, irrigated and re-positioned. Thus, evidence to indicate that additional washings/irrigations still yield a bioactive surface is quite important in the overall development (productisation) of ideas towards the fabrication of covalently modified bioactive Ti devices. Although there is a pattern of loss of functionalisation with reuse, the modified samples were still much better than control metals at stimulating D3-induced MG63 maturation. It is likely therefore that the methodology we have adopted in generating lipid-functionalised Ti could withstand the rigors of arthroplasty, although this will necessitate a far more comprehensive study utilising the appropriate in vivo models in the future. Recently, it has emerged that human, bone marrow-derived stromal cells (hBMSCs) express LPA receptors and that exposure of these cells to LPA can lead to osteoblast formation and resultant matrix mineralisation (Liu et al., 2010, Mansell et al., 2011). The finding that hBMSCs can differentiate into osteoblasts in response to LPA would indicate that LPA-functionalised Ti may indeed be a choice substrate for achieving enhanced osteogenesis at the implant surface.

\section{Conclusions}

The research presented is the first to describe the fabrication of covalent lipid-functionalised Ti surfaces that are better suited to support D3-induced human osteoblast maturation compared to unmodified metal. These studies provide an important first step in realising the development of superior, next-generation, functional biomaterials. What awaits is a study examining the potential of our materials to promote osteoblastogenesis from mesenchymal stem cells and, ultimately, the application of these devices to ascertain their efficacy for improving host cell responses conducive to enhancing osseointegration, performance and longevity.

\section{Acknowledgements}

The authors hereby acknowledge support from the International Team for Implantology (ITI, Basel, Switzerland, grant 548 2008), the National Institute for Health Research (NIHR, i4i stream 1 feasibility study, II-FS-0510-15049) and to the North Bristol NHS Trust (UK) Small Grant Scheme Award (RD25) for their research funding. This article presents independent research commissioned by NIHR under "Titanium Biofunctionalisation". The views expressed in this article are those of the authors and not necessarily those of the NHS, the NIHR or the Department of Health. The authors wish to express their gratitude to Dr. Caroline Taylor, Diabetologia, University of Bristol, for her invaluable help in preparing the figures presented, and the Interface Analysis Centre at the University of Bristol for their help with XPS and SIMS. We would also like to thank Dr. Terje Sjöström of BioMEG, University of Bristol, for the SEM images presented (Fig 7) and to Dr. Mike Whitehouse for valuable discussions during the course of the study. A patent of the study described has since been filed (GB1108390.4). The authors report no conflict of interest.

\section{References}

Aronin CP, Sefcik LS, Tholpady A, Sadik KW, Tholpady SS, Macdonald TL, Peirce SM, Wamhoff BR, Lynch KR, Ogle RC, Botchwey EA (2010a) FTY720 promotes local microvascular network formation and regeneration of cranial bone defects. Tissue Eng Part A 16: 1801-1809.

Aronin CEP, Shin SJ, Naden KB, Rios PD Jr., Sefcik LS, Zawodny SR (2010b) The enhancement of bone allograft incorporation by the local delivery of the sphingosine 1-phosphate receptor targeted drug FTY720. Biomaterials 31: 6417-6424.

Bachle M, Kohal RJ (2004). A systematic review of the influence of different titanium surfaces on proliferation, differentiation and protein synthesis of osteoblast-like MG63 cells. Clin Oral Implants Res 15: 683-692.

Bessho K, Carnes DL, Cavin R, Chen HY, Ong JL (1999) BMP stimulation of bone response adjacent to titanium implants in vivo. Clin Oral Implants Res 10:212218.

Beutner R, Michael J, Schwenzer B, Scharnweber D (2010) Biological nano-functionalization of titanium-based biomaterial surfaces: a flexible toolbox. J R Soc Interface 7: S93-S105.

Blackburn J, Mansell JP (2012) The emerging role of lysophosphatidic acid (LPA) in skeletal biology. Bone 50: 756-762. 
Boyne P, Jones SD (2004) Demonstration of the osseoinductive effect of bone morphogenetic protein within endosseous dental implants. Implant Dent 13: 180-184.

Brinkmann V, Billich A, Baumruker T, Heining P, Schmouder R, Francis G, Aradhye S, Burtin P (2010) Fingolimod (FTY720): discovery and development of an oral drug to treat multiple sclerosis. Nat Rev Drug Discov 9: 883-897.

Calvano CD, Jensen ON, Zambonin CG (2009) Selective extraction of phospholipids from dairy products by micro-solid phase extraction based on titanium dioxide microcolumns followed by MALDI-TOF-MS analysis. Anal Bioanal Chem 394: 1453-1461.

Choi JW, Gardell SE ,Herr DR ,Rivera R, Lee C-W, Noguchi K, Teo ST, Yung YC, Lu M, Kennedy G, Chun J (2011) FTY720 (fingolimod) efficacy in an animal model of multiple sclerosis requires astrocyte sphingosine1phosphate receptor 1 (S1P1) modulation. Proc Natl Acad Sci USA 108: 751-756.

Clemens TL, Karsenty G (2011) The osteoblast: An insulin target cell controlling glucose homeostasis. J Bone Miner Res 26: 677-680.

Clover J, Gowen M (1994) Are MG-63 and HOS TE85 human osteosarcoma cell lines representative models of the osteoblastic phenotype? Bone 15: 585-591.

Ducy P (2011) The role of osteocalcin in the endocrine cross-talk between bone remodelling and energy metabolism Diabetologia 54: 1291-1297.

Dziak R, Yang BM, Leung BW, Li S, Marzec N, Margarone J, Bobek L (2003) Effects of sphingosine-1phosphate and lysophosphatidic acid on human osteoblastic cells. Prostaglandins Leukot Essent Fatty Acids 68: 239249.

Elmengaard B, Bechtold JE, Soballe K (2005a) In vivo study of the effect of RGD treatment on bone ongrowth on press-fit titanium alloy implants. Biomaterials 26: 35213526.

Elmengaard B, Bechtold JE, Søballe K (2005b) In vivo effects of RGD-coated titanium implants inserted in two bone-gap models. J Biomed Mater Res 75A: 249-255.

Ganeva D, Antonietti M, Faul, CFJ, Sanderson RD (2003a) Polymerization of the organized phases of polyelectrolyte-surfactant complexes. Langmuir 19: 65616565.

Ganeva D, Faul CFJ, Gotz C, Sanderson RD (2003b) Directed reactions within confined reaction environments: Polyadditions in polyelectrolyte-surfactant complexes. Macromolecules 36: 2862-2866.

Gidley J, Openshaw S, Pring ET, Sale S, Mansell JP (2006) Lysophosphatidic acid cooperates with 1alpha,25(OH)2D3 in stimulating human MG63 osteoblast maturation. Prostaglandins Other Lipid Mediat 80: 46-61.

Grey A, Chen Q, Callon K, Xu X, Reid IR, Cornish J (2002) The phospholipids sphingosine-1-phosphate and lysophosphatidic acid prevent apoptosis in osteoblastic cells via a signaling pathway involving Gi proteins and phosphatidylinositol-3 kinase. Endocrinology 143: 4755 4763.

Hanawa T (2011) A comprehensive review of techniques for biofunctionalization of titanium. J Periodontal Implant Sci 41: 263-272.
Ikeguchi Y, Nakamura H (2000) Selective enrichment of phospholipids by titania. Anal Sci 16: 541-543.

Kantlehner M, Schaffner P, Finsinger D, Meyer J, Jonczyk A, Diefenbach B. Nies B, Holzemann G, Goddman SL, Kessler H (2000) Surface coating with cyclic RGD peptides stimulates osteoblast adhesion and proliferation as well as bone formation. Chembiochem 1: 107-114.

Kurtz S, Ong K, Lau E, Mowat F, Halpern M (2007) Projections of primary and revision hip and knee arthroplasty in the United States from 2005 to 2030. J Bone Joint Surg Am 89: 780-785.

Larsen MR, Thingholm TE, Jensen ON, Roepstorff P, Joergensen TJD (2005) Highly selective enrichment of phosphorylated peptides from peptide mixtures using titanium dioxide microcolumns. Mol Cell Proteom 4: 873886.

Liu Y, de Groot K, Hunziker EB (2005) BMP-2 liberated from biomimetic implant coatings induces and sustains direct ossification in an ectopic rat model. Bone 36: $745-757$.

Liu Y, Huse RO, de Groot K, Buser D, Hunziker EB (2007). Delivery mode and efficacy of BMP-2 in association with implants. J Dent Res 86: 84-89.

Liu Y-B, Kharode Y, Bodine PVN, Yaworsky PJ, Robinson JA, Billiard J (2010) LPA induces osteoblast differentiation through interplay of two receptors: LPA1 and LPA4. J Cell Biochem 109: 794-800.

Lutz R, Srour S, Nonhoff J, Weisel T, Damien CJ, Schlegel KA (2010) Biofunctionalization of titanium implants with a biomimetic active peptide (P-15) promotes early osseointegration. Clinical Oral Implants Research 21: 726-734.

Mansell JP, Farrar D, Jones S, Nowghani M (2009a) Cytoskeletal reorganisation, 1a,25-dihydroxy vitamin D3 and human MG63 osteoblast maturation. Mol Cell Endo 305: 38-46.

Mansell JP, Shorez D, Farrar D, Nowghani M (2009b) Lithocholate: A promising non-calcaemic calcitriol surrogate for promoting human osteoblast maturation upon biomaterials. Steroids 74: 963-970.

Mansell JP, Barbour M, Moore C, Nowghani M, Pabbruwe M, Sjostrom T, Blom AW (2010) The synergistic effects of lysophosphatidic acid receptor agonists and calcitriol on MG63 osteoblast maturation at titanium and hydroxyapatite surfaces. Biomaterials 31: 199-206.

Mansell JP, Nowghani M, Pabbruwe M, Paterson IC, Smith AJ, Blom AW (2011) Lysophosphatidic acid and calcitriol co-operate to promote human osteoblastogenesis: Requirement of albumin-bound LPA. Prostaglandins Other Lipid Mediat 95: 45-52.

P'ebay A, Bonder CS, Pitson SM (2007) Stem cell regulation by lysophospholipids. Prostaglandins Other Lipid Mediat 84: 83-97.

Schliephake H, Scharnweber D, Dard M, Rossler S, Sewing A, Meyer J, Hoogestraat D (2002) Effect of RGD peptide coating of titanium implants on periimplant bone formation in the alveolar crest. An experimental pilot study in dogs. Clin Oral Implants Res 13: 312-319.

Stenport VF, Roos-Jansaker AM, Renvert S, Kuboki Y, Irwin C, Albrektsson T, Claffey N (2003) Failure to induce supracrestal bone growth between and around partially 
inserted titanium implants using bone morphogenetic protein (BMP): an experimental study in dogs. Clin Oral Implants Res 14: 219-225.

Tatakis DN, Koh A, Jin L, Wozney JM, Rohrer MD, Wikesjo UM (2002) Peri-implant bone regeneration using recombinant human bone morphogenetic protein-2 in a canine model: a dose-response study. J Periodontal Res 37: 93-100.

Tigyi G, Parrill AL (2003) Molecular mechanisms of lysophosphatidic action. Prog Lipid Res 42: 498-526.

Vickers KC, Castro-Chavez F, Morrisett JD (2010) Lyso-phosphatidylcholine induces osteogenic gene expression and phenotype in vascular smooth muscle cells. Atherosclerosis 211: 122-129.

Whyte MP (2010) Physiological role of alkaline phosphatase explored in hypophosphatasia. Ann NY Acad Sci 1192: 190-200.

Wikesjö UM, Sorensen RG, Kinoshita A, Wozney JM (2002) RhBMP-2/alphaBSM induces significant vertical alveolar ridge augmentation and dental implant osseointegration. Clin Implant Dent Relat Res 4: 174-182.

Wikesjö UM, Huang YH, Xiropaidis AV, Sorensen RG, Rohrer MD, Prasad HS, Wozney JM, Hall J (2008) Bone formation at recombinant human bone morphogenetic protein-2-coated titanium implants in the posterior maxilla (type IV bone) in non-human primates. Clin Periodontol 35: $992-1000$.

\section{Discussion with Reviewers}

Reviewer II: Do the authors think that a 3D culture is sufficient to assess the effect of a support on cell maturation?

Authors: The structure of the porous Ti is representative of the surface finish of uncemented implants and is therefore a realistic and valid model. Importantly the data presented do demonstrate that lipid-functionalised porous $\mathrm{Ti}$ is a superior medium for supporting D3-induced osteoblast maturation compared to unmodified metal.

Reviewer II: What are the shear stress values generated when placing a hip implant during arthroplasty? Have you tested the resilience of your coatings under similar conditions?

Authors: This is a valuable point but interestingly shear stress values have not been obtained for the implantation process itself. However, as our functionalisation is for potential cementless fixation of titanium stems we should consider the shear stress value for the cancellous (trabecular) bone within which the stem is inserted. Cancellous bone has a rather low shear stress value of approximately $7 \pm 1.5 \mathrm{MPa}$. We have tethered LPA to the titanium surface via silicon. For LPA to become detached from the metal you would need to sever either a siliconcarbon bond, a silicon-oxygen bond or a titanium-oxygen bond, forces which are many orders of magnitude greater than the shear stress to failure of bone.
Reviewer II: What effect does the functionalisation have on the activity of the molecule compared to the nonfunctionalised form?

Authors: Put very simply we do not have an answer for this. However, from the data presented fixing LPA to the titanium surface still yields a bioactive molecule. Furthermore, we know from the information gleaned for the "recycling" experiments that tethered LPA continues to exhibit functionality. Interestingly, the discovery of LPA as the simplest signalling lipid came from serendipitous observations reporting on a factor(s) associated with serum albumin; albumin is naturally and firmly "functionalised" by LPA and we too found that the ability of D3 to induce human osteoblast maturation was dependent on LPA bound to serum albumin. With this in mind, the bulk of serum LPA is albumin-bound and this perhaps is testimony for LPA being an efficacious signalling factor when part of a much larger substrate. However, there have been no reports detailing whether there is a gain or loss in LPA functionality when attached to the different natural carries, i.e., gelsolin and albumin or indeed agarose and in our case titanium.

Reviewer II: How stable is the siloxanisation technique to hydrolysis when exposed to aqueous (physiological) conditions? A study by Xiao et al. (1998) has shown that $50 \%$ is hydrolysed over the first week and a gradual loss occurs thereafter.

Authors: We used anhydrous solvents to ensure that the titanium surface modification would not be susceptible to functional group losses through hydrolysis. With regard to bond stability in a tissue culture setting however, the information gleaned from the "reuse" experiments are quite useful here (Fig. 9); although there is a pattern of loss of functionality with increasing reuse of the LPAfunctionalised discs this is not mirrored for the FHBPfunctionalised discs. Since the procedure to modify these titanium surfaces are the same, the differential changes observed in biofunctionality are unlikely to reflect any differences in the stability of the lipid-titanium tether. Furthermore, each of the different functionalised discs were subjected to exactly the same number of days (9) in an aqueous environment. We suspect that the differences in MG63 maturation observed for the two different titanium surfaces could be attributed to hydrolysis of the polar head group of LPA by liberated lipid phosphate phosphatases (LPPs). Because the FHBP lacks a head group phosphate it is predicted to be more stable to LPPs (Xu et al., 2005).

\section{Additional Reference}

Xu Y, Aoki J, Shimizu K, Umezu-Goto M, Ham K, Takanezawa Y, Yu S, Mills GB, Arai H, Qian L, Prestwich GD. (2005) Structure-activity relationships of fluorinated lysophosphatidic acid analogues. J Med Chem 48: 33193327. 